\title{
Methodological heterogeneity in dental fluorosis investigations: a critical review
}

\author{
Mohammad Hossein Khoshnevisan, ${ }^{a, b}$ Mohammad Reza Nokhostin, ${ }^{c}$ Behrooz Namvar, ${ }^{b}$ \\ Seyed Hadi Sajjadi, ${ }^{\text {d Mina Pakkhesala,e }}$
}

\author{
aPreventive Dentistry Research Center, Research Institute of Dental Sciences, Shahid Beheshti University of Medical Sciences, Tehran, Iran. \\ ${ }^{b}$ Community Oral Health Department School of Dentistry, Shahid Beheshti University of Medical Sciences, Tehran, Iran. \\ cRestorative Dentistry Department, School of Dentistry, Shahid Beheshti University of Medical Sciences, Tehran, Iran. \\ dOrthodontics Department, Dental Branch, Islamic Azad University of Medical Sciences, Tehran, Iran.

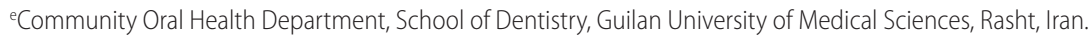 \\ Correspondence to Mina Pakkhesal (email: m_pakkhesal@yahoo.com). \\ (Submitted: 25 February 2017 - Revised version received: 22 March 2017 - Accepted: 13 May 2017 - Published online: 26 September 2017)
}

\begin{abstract}
Objectives The purpose of this investigation was to conduct a comprehensive and critical review of pertinent literature concerning dental fluorosis in order to examine the methodology used in different studies and report the potential sources of heterogeneity among identified reports.

Methods The PubMed database was searched using Medical Subject Headings (MeSH) for articles in English language, published between January 2000 and December 2016. The search was limited to human studies and having abstract available. Subsequently, potential sources of heterogeneity were categorized.

Results Through PubMed search, a total of 56 articles were selected out of 2369 initially identified papers. Subsequently, 17 out of the 56 articles were excluded due to being irrelevant or no full text available. Likewise, genetic studies, in-vitro reports and those studies with no evidence of association were excluded as well. Therefore, 39 remaining articles were critically analyzed in order to identify the potential sources of heterogeneity in dental fluorosis studies. Quality assessment of the reviewed papers demonstrated the following categories as potential sources of heterogeneity in dental fluorosis investigations: "Different methods of fluoride exposure by children", "Different characteristics of study samples under investigation", "Methodological flaws in the fluorosis research design".

Conclusion Given the existing methodological heterogeneity, a consensus development is highly crucial for the standard diagnosis of fluorosis and improvement in homogeneity in future fluorosis investigations globally.

Keywords dental, enamel, fluorosis, dental fluorosis, methodology
\end{abstract}

\section{Introduction}

Tooth decay is a major public health problem affecting a great number of adults and children worldwide. Untreated decay may cause pain, infection, leading mostly to extensive tooth loss in many low- and middle-income countries or disadvantaged communities in high-income countries. Fluoride is an essential nutrient involved in mineralization of teeth and bones. It especially plays important role in tooth remineralization and prevention of tooth decay.

Fluoride is still considered the best protection against dental caries. Water fluoridation was named as 1 of the 10 most important public health measures of the $20^{\text {th }}$ century by the U.S. Centers for Disease Control and Prevention (CDC). ${ }^{2}$

In 2007, WHO World Health Assembly passed resolution WHA 60/16 to enforce universal fluoride access for caries prevention as part of the basic right for human health. ${ }^{3}$ There are three basic fluoride delivery methods for caries prevention; community wide (salt, milk and water fluoridation); professionally applied (fluoride gel, varnish) as well as self-administered (toothpastes and mouth-rinses) methods. ${ }^{1}$

Although, there are some anti fluoridation activities, most claims were unsubstantiated. More than 3000 studies or research papers have been published on fluoride or fluoridation subjects. Based on available reports, the overwhelming weight of the evidence supports the safety and effectiveness of this preventive public health practice. ${ }^{4}$

Some studies have reported that, caries prevention effect of fluoride has been accompanied with very mild to mild fluorosis due to steady exposure to fluoride. This change in the appearance of tooth enamel, is caused by subsurface porosity as a result of hypoplasia or hypo-mineralization that take place during tooth development period. ${ }^{1}$ Although, fluoride's effect may be different in various stages of enamel formation, its effect is maximized when exposure occurs during all stages of tooth formation. ${ }^{5}$ The severity of dental fluorosis depends on dosage of fluoride, duration and timing of fluoride exposure. ${ }^{6}$

Teeth are most susceptible to developmental disturbances during the mineralization phase of tooth formation. In general, the permanent dentition is more susceptible to disturbances in mineralization by environmental toxicants and drugs than the primary dentition, most likely as a consequence of its later development. ${ }^{7}$ Fluorosis is less prevalent and less apparent in primary than in permanent teeth. The fluorosis of primary teeth has only short- rather than long-term consequences. Therefore, the major concern about fluorosis is related to the permanent dentition and particularly the maxillary incisors, because they have great cosmetic importance. ${ }^{8}$ Primary-tooth fluorosis may be used as a prognostic factor in the occurrence of fluorosis in the permanent dentition. Therefore, its recognition by the clinician should raise awareness of possible increased risk in permanent dentition. ${ }^{9}$

The occurrence of fluorosis (which was known as mottled enamel) in the primary dentition was first described in 1935 among children in Arizona. ${ }^{9}$ Studies of primary tooth fluorosis have been conducted in certain high water containing fluoride 
areas in Africa and Europe. The results of these studies have demonstrated the widespread occurrence of primary tooth fluorosis in these locations. The primary molars, particularly the primary second molars were most frequently and severely affected. ${ }^{10}$

The goal of any public health administration is to provide optimum amount of fluoride to public in order to maximize caries prevention at the community level. At the same time, according to best practice guidelines, any sources of overexposure should be recognized and eliminated. Recognition of such sources through scientific research is the best logical method in order to identify and control potential sources of fluoride overexposure. Through the initial review of current literature, we recognized several methodological issues in need of special attention by the research community. Therefore, our aim was to get the most out of published papers using a systematic review methodology. In this article, the results of our findings are reported.

\section{Methods}

The PubMed database was searched using the Medical Subject Headings (MeSH) for studies published between the year 2000 and Dec 2016. The search was limited to review articles, English language, and human studies with available abstract. By using $\mathrm{MeSH}$ search, we used the range of keywords found in relevant articles. For example, "Dental Fluorosis" includes the following keywords: "Fluoroses, Dental", "Dental Fluoroses", "Dental Fluorosis", "Mottled Enamel" and "Enamel, Mottled".

After initial evaluation, some articles were excluded for being irrelevant, in vitro animal (mice) studies, reports or unavailable full texts. The remaining articles were critically evaluated in order to synthesize the contemporary understanding on clinical diagnosis of fluorosis, in order to better control the main sources of disorder while, making the best use out of optimum fluoride exposure.

The full texts of included studies were independently evaluated by two review authors. Any disagreements were discussed and a third review author consulted to achieve consensus where necessary.

\section{Results}

The initial electronic search in PubMed identified 56 articles from a total of 2369 studies. (Table 1) Subsequently, 17 out of the 56 articles were excluded due to irrelevance or full text not being accessible, genetic studies and in-vitro reports. Finally, data extraction was conducted from 39 remaining articles. (Fig. 1) Qualitative analyses of the reviewed papers demonstrated the following categories as potential sources of heterogeneity in dental fluorosis investigations: Difference in methods of fluoride exposure by children, Different characteristics of study samples under investigation, Methodological flaws in the fluorosis research design. (Table 2)

\section{Discussion}

The purpose of this investigation was to conduct a comprehensive and critical review of pertinent literature concerning dental fluorosis in order to examine the methodology used in different studies and report the potential sources of heterogeneity among identified reports.

\begin{tabular}{clc}
\hline Table 1. PubMed search strategy & \\
\hline $\begin{array}{c}\text { Search } \\
\text { step }\end{array}$ & Inclusion \& exclusion criteria & $\begin{array}{c}\text { No. of } \\
\text { publications }\end{array}$ \\
\hline 1 & Search dental fluorosis [MeSH Terms] & 2369 \\
2 & $\begin{array}{l}\text { Search dental fluorosis [MeSH Terms] } \\
\text { Filters: Review }\end{array}$ & 194 \\
3 & $\begin{array}{l}\text { Search dental fluorosis [MeSH Terms] } \\
\text { Filters: Review; Abstract }\end{array}$ & 135 \\
4 & $\begin{array}{l}\text { Search dental fluorosis [MeSH Terms] } \\
\text { Filters: Review; Abstract; Publication date } \\
\text { from 2000/01/01 to 2016/12/31 }\end{array}$ & 64 \\
5 & $\begin{array}{l}\text { Search dental fluorosis [MeSH Terms] } \\
\text { Filters: Review; Abstract; Humans }\end{array}$ & 63 \\
6 & $\begin{array}{l}\text { Search dental fluorosis [MeSH Terms] } \\
\text { Filters: Review; Abstract; Humans; English }\end{array}$ & 56 \\
\hline
\end{tabular}

\section{Different Methods of Fluoride Exposure by Children}

Researchers have identified 4 sources of increasing the risk of dental fluorosis as follows: 1) the natural or fluoridated drinking water, 2) fluoride supplements, 3) topical fluoride (especially fluoride toothpastes when ingested during brushing), and formula prescribed for children under 2-years of old. Furthermore, using multiple sources of fluoride such as can-foods, soda, etc., have been found to be an important contribution to daily over-consumption of fluoride. ${ }^{11,12}$

Many studies did not take into account the effect of all risk factors involved or could affect children's risk of tooth decay or dental fluorosis. There was also substantial variation between the results of the studies, many of which took place before the introduction of fluoride toothpaste.

Water fluoridation can be regarded as a low-cost method of fluoride delivery for those communities where oral health care and particularly fluoride dentifrices are not widely available and/or is not affordable, causing reduction in socioeconomic related dental inequalities. ${ }^{13-15}$

Around the world, dental fluorosis has always been regarded as a public health problem in those areas where natural fluoride in the community drinking water exceeds optimal levels. However, residents of optimally fluoridated areas have not been considered to be at risk for dental fluorosis. ${ }^{16}$ The "optimal" water fluoride concentrations can minimize the risk for both dental fluorosis and dental caries vary between 0.7 ppm and $1.2 \mathrm{ppm}$, depending on mean temperature of any given geographical area. ${ }^{17}$ Enamel fluorosis occurs at fluoride levels $(1.8-2.2 \mathrm{mg} / \mathrm{L})$ which is much lower than the skeletal fluorosis becomes clinically evident (20-80 mg per day). ${ }^{14}$

Based on current literature, the fluorosis caused by water fluoridation (40\%) is being less than that attributable to other fluoride sources (60\%). By the 1990s, fluoride toothpaste accounted for $\geq 90 \%$ of the toothpaste market in most economically developed countries, and accidental or intentional ingestion of fluoridated toothpaste especially in children has become a potentially important risk factor for excessive fluoride exposure. ${ }^{18}$ When recent data are compared to historical data, the results seem to indicate a trend toward a higher prevalence of fluorosis. However, due to widespread use of fluoridated products (such as fluoride supplements, fluoride toothpaste, and fluoride in the food and beverages), concern has been expressed in 


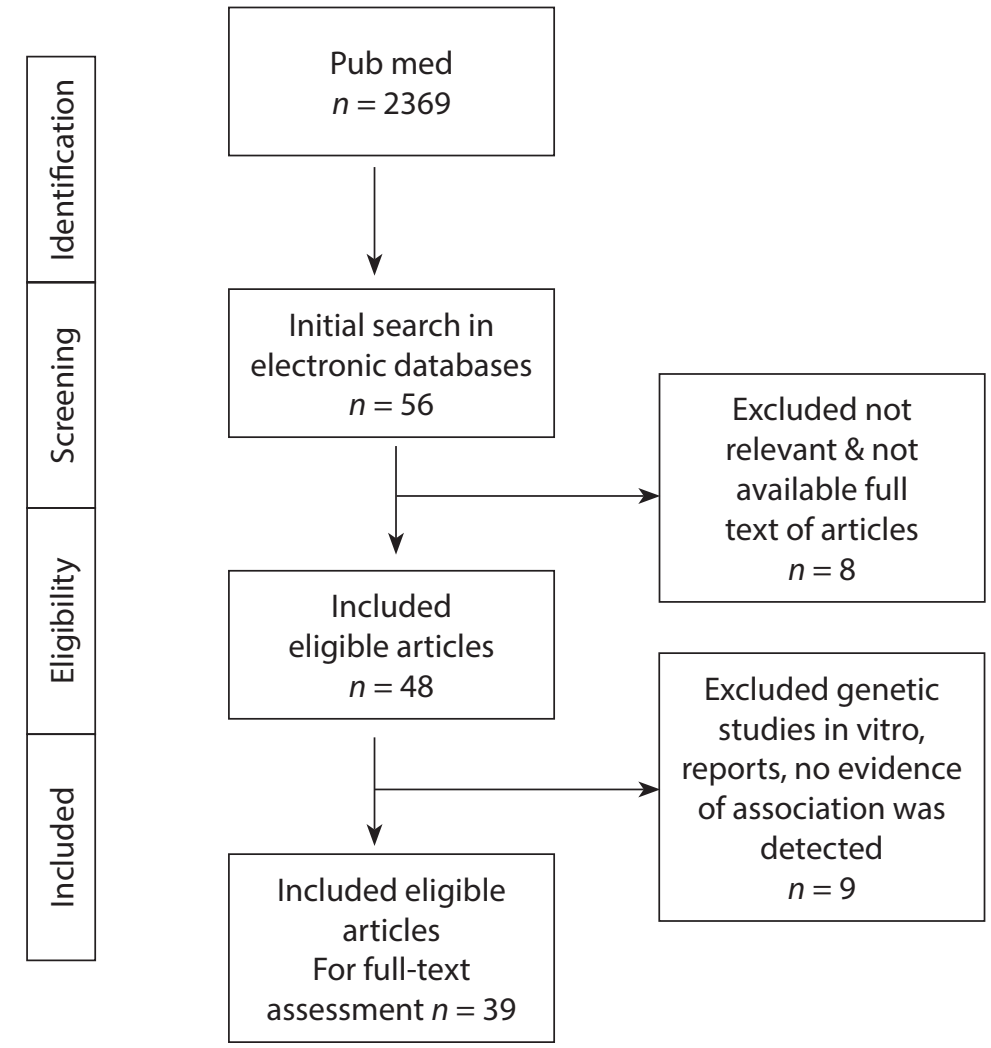

Fig. 1 Flow chart showing search strategy and numbers of included and excluded articles.

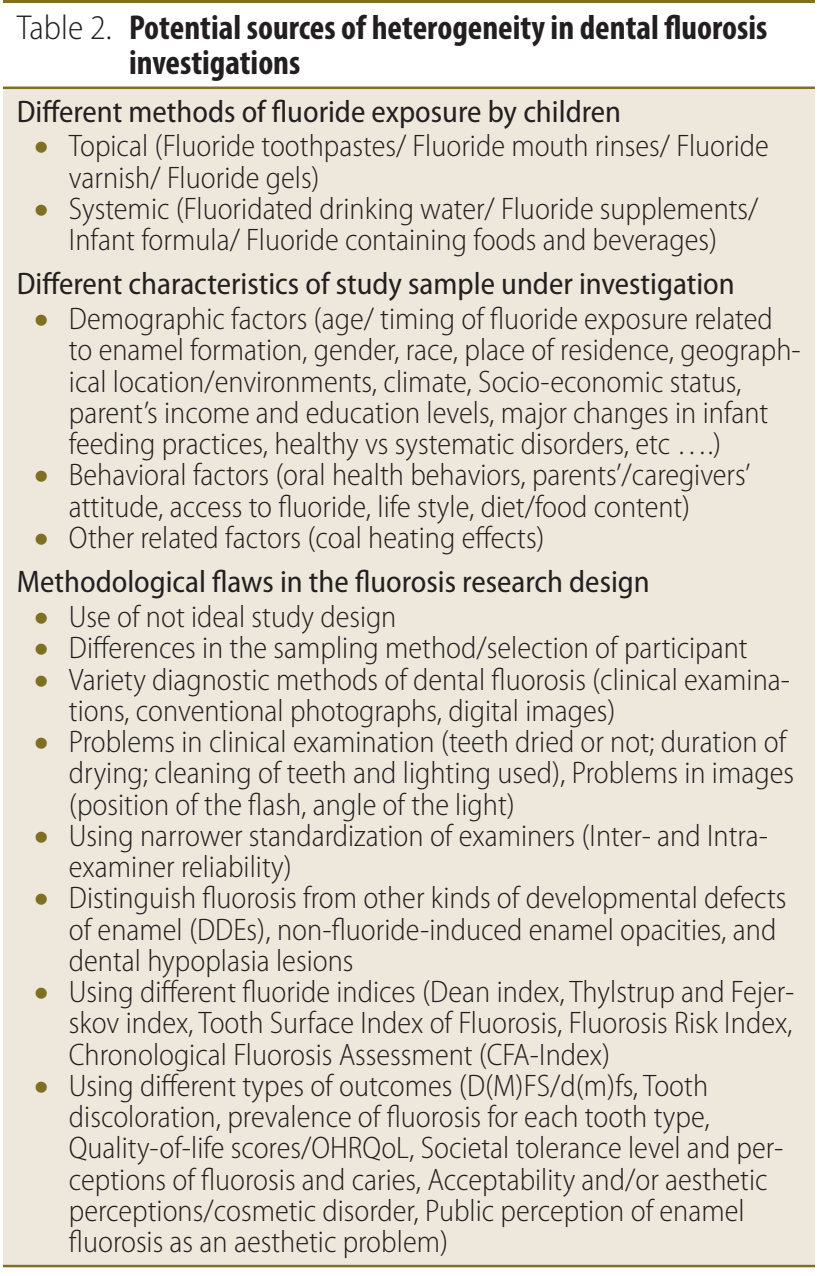

recent years over a possible increase in the prevalence of dental fluorosis worldwide in optimally fluoridated and even suboptimally fluoridated areas. Also, concurrent use of multiple sources of fluoride mainly fluoride intake from meals prepared with fluoridated salt; living at a high altitude; living in a hot climate have been reported. Dental fluorosis was association has been reported with: bottled water, soft drinks, and juices; excessive fluoride in deep well water; boiled water as well. ${ }^{16}$

A higher risk of developing dental fluorosis is expected when excess exposure to ingested topical fluoride in young children occurs during permanent tooth development. Mild dental fluorosis is demonstrated by white lines or streaks visible only to dentists under good lighting in the clinic.

Moreover, infant formula reconstituted with fluoridated water has been reported to be a risk factor for enamel fluorosis when consumed during the period of 13-24 months. ${ }^{10,19}$ The shortest duration of breast-feeding usually corresponded to the longest duration of infant formula use. ${ }^{20}$

Many studies have reported a clear association between fluoride supplements use by children under age 6 and enamel fluorosis. Subjects who used fluoride supplements during the first 6 years of life had a 28 -fold increase in the risk of fluorosis when compared to unexposed subjects. ${ }^{10}$ It is important that, individual water sources be tested for fluoride level before prescribing or recommending fluoride products and suppliant for young children in order to control their fluoride ingestion. ${ }^{17}$

Consistent evidence is available that shows fluoride tablets used during the first 3 years of life increased the risk of developing fluorosis; the first year of life appears to be the high risk period for incisors. ${ }^{21,22}$

It is possible that slow dissolution fluoride lozenges may start to play a role in caries control in older children and 
adults, those who are no longer at risk of enamel fluorosis. Therefore, topically "applied" fluoride had dramatic caries reductions (approximately 80\%) when compared to systemic fluoride users. Thus, chewable fluoride supplements can provide caries protective benefit in children and adults when the overall conditions are considered. The decision-making on the fluoride supplements prescription to children ought to be delegated on dentists and primary care pediatricians and by no means it should be taken as a common preventive measure in the pediatric population. ${ }^{23}$

\section{Different Characteristics of Study Samples Under Investigation}

Individuals, families and communities may differ in their fluoride exposure level. ${ }^{1}$

\section{Demographic Factors}

The most critical period for fluorosis in the permanent dentition is considered to be during the latter stages of pre-eruptive tooth development; for the anterior permanent teeth which is between age 22 and 25 months old. ${ }^{8,24-26}$ The fluorosis can be used as a biomarker for the level of fluoride exposure in populations during the time of enamel formation. ${ }^{22,27}$

Moreover, subjects living in a middle income households who had used fluoride supplements through the first six years of life demonstrated 28-fold increase in the risk of fluorosis compared with unexposed subjects in the lower median income households. ${ }^{23}$ Interestingly, children from high socioeconomic group place approximately $24 \%$ more dentifrice on their toothbrush than their counterparts from lower socioeconomic classes. ${ }^{24}$

When considering socio-environmental, health context and especial demographic characteristics of a given population group, the production of epidemiological data on fluorosis should allow an understanding of the health and disease situation, even though offering limited conditions for the comparability of findings. ${ }^{28}$

The relationship between water fluoridation and social inequalities can be evaluated by the Human_Development Index (HDI). This is particularly important for developing countries where water fluoridation is feasible. ${ }^{29,30}$

Other variables that have been associated with an increased risk of fluorosis are: socio-demographic variables such as the child's age, gender, and race; parent's income and education levels; feeding practices such as weaning before 9 months of age and breast feeding period; and fluoride mouth-rinse. It has been reported that, male Caucasian children, with high income and educational level parents were more likely to have fluorosis. ${ }^{27}$ Furthermore, the increased prevalence of fluorosis in black non-Hispanics may suggest a genetic influence on fluorosis susceptibility. ${ }^{12}$

The severity of dental fluorosis depends on when and for how long the overexposure to fluoride occurs, the individual response, weight, degree of physical activity, nutritional factors and bone growth, suggesting that similar dose of fluoride may lead to different levels of dental fluorosis. Other factors that may increase the individual susceptibility to dental fluorosis are altitude, malnutrition and renal insufficiency. ${ }^{11,27}$ Fluorosis begins with the exposure of the tooth bud to high concentrations of fluoride ion during its formation. Other factors such as low body weight, skeletal growth rate, and periods of bone remodeling also affect the severity of this condition. ${ }^{28}$

Epidemiological studies performed in the countries of Kenya and Tanzania have indicated that higher prevalence and severity of fluorosis may be related to high altitude, even when suboptimal concentrations of fluoride are present in the drinking water. ${ }^{16}$

\section{Behavioral Factors}

The increase in level of enamel fluorosis in permanent incisors was attributed to increased use and swallowing of fluoride toothpaste by infants and children. In some countries like Australia, Canada, and Republic of Ireland, it has led in reduction of fluoride levels in drinking water. ${ }^{22}$

Fluoride ingested from toothpaste was correlated with tooth-brushing habits, frequency of brushing, frequency of rinsing, post brushing rinsing behavior, fluoride concentration in dentifrices, amount of toothpaste dispensed (the diameter of the orifice of the tubes, the length of the head of the toothbrush, the flavor and attractive visual characteristics of the dentifrice, weight of toothpaste used), as well as child's body weight of the child. ${ }^{10,12,18,24,31,32}$ Parental supervision is necessary for placement of toothpaste and every time children should be reminded to limit the amount of toothpaste used until age 6-7 years old. This may be due to parental brushing for their babies with toothpaste at too young age (before 24 months) or when children have not learned how to adequately rinse out their mouths and they, ingest much of toothpaste while brushing their teeth or rinsing their mouth. ${ }^{10}$ Healthcare professionals play an important role in explaining to parents and/or caregivers about the risks and benefits of fluoridated dentifrices used for children. Moreover, if the risk of fluorosis is of concern, the fluoride level of toothpaste for young children is recommended to be not lower than 1000 ppm. ${ }^{25}$ However, improper use of fluoride toothpaste in children under 6 can increase the risk of fluorosis. ${ }^{33}$

Another factor that may contribute to the amount of dentifrice ingested could be the quantity of detergent present in toothpaste formulation. Because dentifrices with a low detergent, generate less foam during brushing, and as a result, it poses smaller risk of ingestion. ${ }^{24}$

Besides, motivated parents would probably be more involved in controlling the oral hygiene habits of their children, which may lead to increased fluoride exposure. ${ }^{18}$ Also, "preventionism" behavior, as defined by the irrational use of fluoride in populations with more access to goods and services may be implicit in the genesis of some iatrogeny. ${ }^{28}$

The majority of foods assessed for their fluoride level are those meant for infants and young children. Seafoods have high concentration of fluoride. Also, processing foods with fluoridated water typically have higher fluoride concentrations than foods processed with non-fluoridated water. Likewise, processing method of infant cereals has been shown to affect their fluoride concentration. ${ }^{17}$ The ingestion of fluoride may be delayed depending on the type of food present in the stomach. ${ }^{24}$ The major inhibitor of fluoride absorption is calcium. The presented data indicate that, now the calcium intakes by infants are less than in the 1930 s to 1960 s period. In addition, the considerable increase in consumption of soft drinks and fruit juices by children during the past 20 years has been associated with 
decreased milk consumption and a consequent decrease in calcium intake. Thus, bioavailability of fluoride in the diets of infants and children is probably greater now than before.

The increase in percentage of communities with fluoridated water has resulted in an increase in the mean content of fluoride consumed, not only in soft drinks and fruit juices, but in canned goods, leading to increased intake of fluoride by individuals and communities with non-fluoridated water. Furthermore, tea is regularly consumed by a minority of children in the United States, and because of its high fluoride concentration (commonly $3 \mathrm{mg} / \mathrm{L}$ ), can contribute substantially to the fluoride intakes of these children.

\section{Other Related Factors}

Endemic fluorosis is a public health concern in China due to excessive consumption of fluoride in drinking water, brick tea, food that was contaminated by indoor coal burning, and inhalation of coal burning air. Also, the high-fluoride spring water was a possible source of fluorosis. ${ }^{34}$ Additionally, due to the mobilization of fluoride by the burning of mineralized coals in unvented or poorly vented stoves, millions of people in Guizhou suffer from dental and skeletal fluorosis. Unhygienic living environments and unhealthy lifestyles can promote endemic fluorosis. Therefore, multiple factors, including geographical location, humid climate, the geological and geochemical environment, economics, educational level, drying and storing methods of foodstuffs, unhealthy use of coal in the household as well as traditional lifestyles may contribute to incidence in endemic fluorosis. ${ }^{34}$

\section{Methodological Flaws in the Fluorosis Research Design}

\section{Study design and control of bias}

Concern for the increase in the prevalence of dental fluorosis led to studies designed to identify the various risk factors for fluorosis. While a few studies were case-control, most others were cross-sectional in design. This (cross-sectional) study design is not an ideal method for assessing risk indicators or risk factors. Hence, this design has been used in some fluorosis literature. Another major criticism of most fluorosis studies is the use of retrospective assessment of fluoride exposures, due to inherent recall bias in such design. Although, the recall bias should be assumed as random, and therefore not overly affecting the results. ${ }^{27}$

\section{Sampling Method}

The Brazilian literature shows differences in the sample planning, sample size, age bracket, inter-examiner reliability, reproducibility, health surveillance data accuracy, and territorial base. ${ }^{28}$

Furthermore, the ages of the study participants in some of the studies were not appropriate to assess the research question. Some studies used children between 6 and 13 years old. Six-year-old children do not have many erupted permanent teeth yet. This sample may have underestimated the prevalence of fluorosis. ${ }^{27}$

The dental fluorosis prevalence study in Mexico showed two issues: (1) Researchers did not record the place of residence for their participants at the time of oral examination. (2) Also, researchers failed to verify the duration of stay in place of residence from birth to age 6 years for the study participants. As a result, it is not possible to determine if the fluorosis observed in these samples was a true reflection of the communities where they lived. Bias could have been introduced if some participants were not permanent residents of those communities under investigation. Also, adding to the question about the representativeness of the samples due to the fact that many of the studies were used convenient samples. ${ }^{16}$

Another limitation was related to the small sample size as a consequence of limited funding; adequate number of subjects could be recruited for evaluation, if there was no financial constraints. ${ }^{18}$

\section{Diagnostic Methods of Dental Fluorosis}

One major factor that limits the comparability of epidemiologic studies on enamel defects is related to a variety of diagnostic methods used. Dental fluorosis has several fluorosis-specific criteria used for its diagnosis. Studies of the diagnosis of dental fluorosis can be affected by a large number of factors, such as examiner bias, intra-examiner and inter-examiner reliability, examiner drift, index validity due to varying methodologies (teeth dried or not; duration of drying; cleaning of teeth and lighting used). ${ }^{35}$

In epidemiological studies of dental fluorosis, the photographic method offers the following advantages: images can be read by trained examiners, blinded to the fluoridation status of the subject; images can be double-scored and cross-checked with the development of consensus in divergent scores. Images are permanent record of the enamel, and are useful for measuring changes in enamel over time. ${ }^{35}$ The use of a standard photographic technique may be helpful in controlling potential discrepancies and can better reflect the reality. ${ }^{36}$ A technique employing standardized film, handling procedures, camera equipment, exposure time, lighting conditions, drying time of teeth, camera angulation, lip retraction and processing procedures has been developed and employed successfully in seven EU countries and in the National Survey of Children's Oral Health in Ireland. ${ }^{37}$ Therefore, an experienced and/or trained examiner is necessary when taking standardized photos in order to minimize both specular reflection and lip shadow. The only potential disadvantage of using photography method is the increase in the cost of the study.

Despite the advances in digitalized imaging, conventional photographs are more often used in epidemiological investigations. The digital camera offers the following advantages: It maintains confidentiality, as it can photograph the teeth alone; measurements of variation in density are possible with digital images; greater resolution increases the definition of the image; and images can be easily stored in digital systems. For using digital camera, one should be well versed and experienced with the equipment. In this case, the investigators have to account for the cost of equipment. ${ }^{35}$

The results suggest inter-examiner reliability is greater and fluorosis scores higher when using the photographic method compared to clinical examinations. ${ }^{38-40}$ Examiners training has been reported in many collected studies on dental fluorosis prevalence in Mexico. However, the measurements of calibration were rarely reported. Fluorosis is a difficult condition to diagnose, even for the experienced examiner. The lack of information on accuracy of the examiners, who were determining fluorosis status, makes it difficult to know whether differences 
reported among regions or in time trends as were a true reflection of the population differences in fluorosis prevalence or they were instead a reflection of differences in the examiners assessments. ${ }^{16}$

\section{Distinguish Fluorosis from Other Kinds of Developmental Enamel Defects}

It should be noted that fluorosis is not the only type of disturbance found in dental enamel; enamel opacities can result from number of causes unrelated to fluoride use. The differential diagnosis between fluorosis and non-fluorideinduced opacities should be established based on symmetrical and asymmetrical and/or discrete patterns of opaque defects. These criteria imply that all symmetrically distributed and non-discrete opaque conditions of enamel are considered fluorosis. It's important to emphasize that non-fluoride enamel opacities include all categories of opacities not defined as fluorosis, i.e. dental hypoplasia lesions that are commonly characterized as discrete, demarcated white or discolored opacities often affecting a single tooth and, less frequently, multiple teeth, with a symmetrical distribution, and result from a wide variety of systemic or local factors. ${ }^{11}$

\section{Using Different Fluoride Indices}

In relation to fluorosis publications, the first major difficulty relates to the comparability of the studies, due to the diversity of indices used. In 1942, H.T. Dean developed an index for description and diagnosis of enamel fluorosis. This classification is still the 'gold standard', though other indices have been developed including the widely used Thylstrup and Fejerskov Fluorosis Index (TFI). ${ }^{12,41}$

Dean's Index scores the two teeth that are most affected. The Dean index even though describing the severity of fluorosis with less variation, is widely used, and is the index recommended by the World Health Organization (WHO), since it can be used safely in public health studies. Thylstrup et al. proposed a modification of Dean's index known as the TF index. This classifies clinical features of fluorosis that reflect histopathological changes following histological examination using ordinary and polarized light on affected enamel. The index requires the teeth to be dried before examination, which gives better precision, so it is recommended for populations with a higher prevalence of the disease. Other indices are used less frequently, since they are not conducive to comparative approaches. ${ }^{28}$ Tooth Surface Index of Fluorosis (TSIF) described by Horowitz et al. provides an analysis based on aesthetic concerns and examines teeth when wet. The Fluorosis Risk Index (FRI), developed by Pendrys, is designed to produce an accurate association between age- specific exposures to fluoride and the development of fluorosis. It divides the enamel surface of the permanent teeth into two developmentally related groups of surface zones. Code 1 began formation during the first year of life and code 2 began during the third to sixth years of life. The scores are recorded for each zone. ${ }^{10}$ In addition; it must be kept in mind that fluorosis prevalence is directly influenced by the case definition used to calculate it. For example, the case definition for the Tooth Surface Index of Fluorosis is based on the tooth surface unit, while Dean's Index defines cases of fluorosis on the basis of individual teeth. ${ }^{16}$ In 1993, Evans developed the Chronological Fluorosis Assessment (CFA) Index to investigate the chronological development of enamel fluorosis. Evans et al. refined the estimated time for enamel fluorosis to occur. ${ }^{10}$

\section{Using Different Types of Outcomes}

Fluorosis as intrinsic discoloration occurs following a change to the structural composition of the dental hard tissues. ${ }^{42}$ The primary outcome was caries increment in the permanent or primary dentition, as measured by the change in decayed, (missing), and filled tooth surfaces $[D(M)$ $\mathrm{FS} / \mathrm{d}(\mathrm{m}) \mathrm{fs}$ ] from baseline.

The other primary outcome measure was the percentage prevalence of fluorosis in the permanent dentition. The timing of the outcome measurement should have been taken when most of the permanent teeth of interest were erupted in the study participants. If available, the prevalence of fluorosis for each tooth type is recorded. ${ }^{25}$

Do and Spencer found reported that, children who had mild fluorosis demonstrated higher quality-of-life scores than those children who had caries or more advanced fluorosis. This research should be expanded to define the societal tolerance level and perceptions of fluorosis and caries. Evidence, rather than our professional perceptions, should guide us to decide what is acceptable by society. ${ }^{43,44}$

Dental fluorosis is not a condition that causes pain or has clinical symptoms. The effects of mild fluorosis are subjective; thus, reports of dental fluorosis prevalence and severity alone do not give enough information to understand the effects at the public health level. To examine the effects of dental fluorosis, the early studies assessed acceptability and/or aesthetic perceptions concerning photographs of cases and/or subjects' teeth with and without dental fluorosis in interested populations. ${ }^{45}$

Studies have shown that the oral region is of primary importance in determining overall facial attractiveness. Also, dental appearance is an important contributor to one's selfperceived body image. Mild fluorosis is seen by dental professionals to be of little cosmetic consequence but to the person involved it may be an aesthetic problem. ${ }^{37}$

Numerous studies have addressed the public perception of enamel fluorosis as an aesthetic problem. Although, these studies were conducted in various countries (Australia, Canada, UK, USA) using different indices of enamel fluorosis, the findings generally from all these studies reflect that, both parents and children are less concerned about low levels of fluorosis than dentists. Children with such low-level fluorosis are less likely to have experienced tooth decay. However, aesthetically objectionable fluorosis is a rare outcome, observed in only about 2 percent of children. ${ }^{14}$

Dental fluorosis has not been identified as a public health problem in North America. However, given the trend toward whitening teeth and the increased demand for cosmetic dentistry, public rejection of even the mildest form of fluorosis could pose problems for dentistry's timetested reliance on this proven and cost-effective caries preventive agent.?

Since dental fluorosis in the United States and other nations without high levels of naturally-occurring fluoride is mild or very mild, with little impact on Oral Health Related Quality of Life (OHRQoL), dental professionals should emphasize on the 
appropriate use of fluoride for its caries prevention benefits as well as preventing moderate/severe fluorosis. ${ }^{45}$

One review study in identifying the risk factors for dental fluorosis has reported that, the reasons for increase in the prevalence of dental fluorosis as reported by different investigations are due to employing various study designs, using different sample populations, many exposed to multiple sources of fluoride, and using different indices to measure fluorosis. As a result not only the conclusions of some of these studies are not similar, but in some cases is confusing and even contradictory. ${ }^{27}$

\section{Conclusion}

Given the existing methodological heterogeneity, a consensus development is highly crucial for the standard diagnosis of fluorosis and improvement in homogeneity in future fluorosis investigations globally.

\section{Conflicts of Interest}

There are no conflicts of interest.

\section{References}

1. Iheozor-Ejiofor Z, Worthington HV, Walsh T, O'Malley L, Clarkson JE, Macey R, et al. Water fluoridation for the prevention of dental caries. Cochrane Database Syst Rev. 2015;(6):CD010856.

2. Levy SM. An update on fluorides and fluorosis. J Can Dent Assoc. 2003; 69:286-291.

3. Petersen PE. World Health Organization global policy for improvement of oral health—World Health Assembly 2007. Int Dent J. 2008:58:115-121.

4. Clark MB, Slayton RL. Section on oral health. Fluoride use in caries prevention in the primary care setting. Pediatrics. 2014;134:626-633.

5. Bronckers AL, Lyaruu DM, DenBesten PK. The impact of fluoride on ameloblasts and the mechanisms of enamel fluorosis. J Dent Res. 2009:88:877-893.

6. Clarkson JJ, McLoughlin J. Role of fluoride in oral health promotion. Int Dent J. 2000;50:119-128.

7. Billings RJ, Berkowitz RJ, Watson G. Teeth. Pediatrics. 2004;113:1120-1127.

8. Fomon SJ, Ekstrand J, Ziegler EE. Fluoride intake and prevalence of dental fluorosis: trends in fluoride intake with special attention to infants. J Public Health Dent. 2000;60:131-139.

9. Warren JJ, Kanellis MJ, Levy SM. Fluorosis of the primary dentition: what does it mean for permanent teeth?. J Am Dent Assoc. 1999;130:347-356.

10. Browne D, Whelton $\mathrm{H}, \mathrm{O}^{\prime}$ Mullane D. Fluoride metabolism and fluorosis. J Dent. 2005;33:177-186.

11. Abanto Alvarez J, Rezende KM, Marocho SM, Alves FB, Celiberti P, Ciamponi AL. Dental fluorosis: exposure, prevention and management. Med Oral Pato Oral Cir Bucal. 2009;14:103-107.

12. Denbesten P, Li W. Chronic fluoride toxicity: dental fluorosis. Monogr Oral Sci. 2011;22:81-96

13. Sampaio FC, Levy SM. Systemic fluoride. Monogr Oral Sci. 2011;22:133-145.

14. Newbrun E. What we know and do not know about fluoride. J Public Health Dent. 2010;70:227-233.

15. Harding MA, O'Mullane DM. Water fluoridation and oral health. Acta Med Acad. 2013:42:131-139.

16. Soto-Rojas AE, Ureña-Cirett $J$, Martínez-Mier EA. A review of the prevalence of dental fluorosis in Mexico. Rev Panam Salud Publica. 2004;15:9-18.

17. Warren JJ, Levy SM. Current and future role of fluoride in nutrition. Dent Clin North Am. 2003;47:225-243

18. Siew Tan B, Razak IA. Fluoride exposure from ingested toothpaste in 4-5-year-old Malaysian children. Community Dent Oral Epidemiol. 2005;33:317-325.

19. Pendrys DG. Risk of enamel fluorosis in nonfluoridated and optimally fluoridated populations: considerations for the dental professional. J Am Dent Assoc. 2000;131:746-755.

20. Hujoel PP, Zina LG, Moimaz SA, Cunha-Cruz J. Infant formula and enamel fluorosis: a systematic review. J Am Dent Assoc. 2009;140:841-854.

21. Buzalaf MA, Levy SM. Fluoride intake of children: considerations for dental caries and dental fluorosis. Monogr Oral Sci. 2011;22:1-19.

22. O'Mullane DM, Baez RJ, Jones S, Lennon MA, Petersen PE, Rugg-Gunn AJ, et al. Fluoride and oral health. Community Dent Health. 2016;33:69-99.

23. Oganessian E, Lencová E, Broukal Z. Is systemic fluoride supplementation for dental caries prevention in children still justifiable?. Prague Med Rep. 2007;108:306-314

24. Ekambaram M, Itthagarun A, King NM. Ingestion of fluoride from dentifrices by young children and fluorosis of the teeth-a literature review. J Clin Pediatr Dent. 2011:36:111-121.

25. Wong MC, Glenny AM, Tsang BW, Lo EC, Worthington HV, Marinho VC. Topical fluoride as a cause of dental fluorosis in children. Cochrane Database Syst Rev. 2010;(1):CD007693
26. DenBesten PK. Biological mechanisms of dental fluorosis relevant to the use of fluoride supplements. Community Dent Oral Epidemiol. 1999:27:41-47

27. Mascarenhas AK. Risk factors for dental fluorosis: a review of the recent literature. Pediatr Dent. 2000;22:269-277.

28. da Cunha LF, Tomita NE. Dental fluorosis in Brazil: a systematic review from 1993 to 2004. Cad Saude Publica. 2006:22:1809-1816.

29. Warren JJ, Levy SM. Systemic fluoride. Sources, amounts, and effects of ingestion. Dent Clin North Am. 1999:43:695-711.

30) Nona Attaran, Mohammad H Khoshnevisan, Zahra Ghorbani, Mina Pakkhesal, Danoosh Dehghanian. Dental caries predictors in countries with different human development index: a review of articles. J Int Oral Health. 2016;8:182-190.

31. Davies RM, Ellwood RP, Davies GM. The rational use of fluoride toothpaste. Int J Dent Hyg. 2003;1:3-8

32. Ellwood RP, Cury JA. How much toothpaste should a child under the age of 6 years use?. Eur Arch Paediatr Dent. 2009:10:168-174.

33. Wong MC, Clarkson J, Glenny AM, Lo EC, Marinho VC, Tsang BW, et al. Cochrane reviews on the benefits/risks of fluoride toothpastes. J Dent Res. 2011;90:573-579.

34. Chen J, Liu G, Kang Y, Wu B, Sun R, Zhou C, et al. Coal utilization in China: environmental impacts and human health. Environ Geochem Health. 2014;36:735-753.

35. Martins CC, Chalub L, Lima-Arsati YB, Pordeus IA, Paiva SM. Agreement in the diagnosis of dental fluorosis in central incisors performed by a standardized photographic method and clinical examination. Cad Saude Publica. 2009;25:1017-1024

36. Sajjadi SH, Khosravanifard B, Moazzami F, Rakhshan V, Esmaeilpour M. Effects of three types of digital camera sensors on dental specialists' perception of smile esthetics: a preliminary double-blind clinical trial. J Prosthodont. 2016;25:675-681.

37. Whelton HP, Ketley CE, McSweeney F, O'Mullane DM. A review of fluorosis in the European Union: prevalence, risk factors and aesthetic issues. Community Dent Oral Epidemiol. 2004;32:9-18.

38. Cruz-Orcutt N, Warren JJ, Broffitt B, Levy SM, Weber-Gasparoni K. Examiner reliability of fluorosis scoring: a comparison of photographic and clinical examination findings. J Public Health Dent. 2012; 72:172-175

39. Soto-Rojas AE, Martínez-Mier EA, Ureña-Cirett J, Jackson RD, Stookey GK. Development of a standardisation device for photographic assessment of dental fluorosis in field studies. Oral Health Prev Dent. 2008:6:29-36.

40. Mohd Nor NA, Chestnuttl IG, Chadwick BL. Examiner reliability in fluorosis scoring: a comparison of photographic and clinical methods. Community Dent Health. 2016:33:145-150.

41. Sabokseir A, Golkari A, Sheiham A. Distinguishing between enamel fluorosis and other enamel defects in permanent teeth of children. Peer J. 2016:4:e1745.

42. Watts A, Addy M. Tooth discolouration and staining: a review of the literature. Br Dent J. 2001;190:309-316.

43. Do LG, Spencer A. Oral health-related quality of life of children by dental caries and fluorosis experience. J Public Health Dent. 2007:67:132-139.

44. Ismail Al, Hasson H. Fluoride supplements, dental caries and fluorosis: a systematic review. J Am Dent Assoc. 2008;139:1457-1468.

45. Chankanka O, Levy SM, Warren JJ, Chalmers JM. A literature review of aesthetic perceptions of dental fluorosis and relationships with psychosocial aspects/oral health-related quality of life. Community Dent Oral Epidemiol. 2010;38:97-109. 